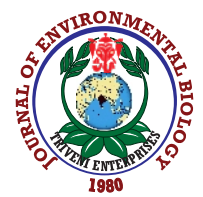

\title{
Color and sugar preferences of adult black soldier fly (Hermetia illucens) (Dipetera: Stratiomyidae) for feeding and oviposition
}

\author{
N. Romano*, H. Fischer and N. Egnew \\ Aquaculture/Fisheries Center, University of Arkansas at Pine Bluff, 1200 North University Drive, Pine Bluff, AR 71601, USA \\ *Corresponding Author Email : romanon@uapb.edu
}

\section{Abstract}

Aim: The aims of this study were to determine the preference of adults $H$. illucens to different sugar types, followed by examining which color background encourages feeding.

Methodology: Three separate experiments were conducted; first, the feeding preference of $H$. illucens adults to three different sugar types were examined. Second, whether six different colors influenced feeding preference. Thirdly, oviposition frequency/clutch weight among two different colors and three bait types.

Results: Honey was more preferred as compared to white sugar, brown sugar or water. A white background was preferred when feeding, while blue color was completely avoided. In contrast, blue was preferred over white for oviposition, based on significantly more clutches and weights. Moreover, baiting $\mathrm{H}$. illucens adults with spent coffee grounds led to significantly more clutches and weights compared to sweet potato or spoiled fish feeds. Thus, using white backgrounds with honey can encourage feeding while a blue background can encourage oviposition.

Interpretation: Feeding and oviposition activities can be easily separated in a simple and cost-effective way for commercial production.

Key words: Clutch weight, Color preference, Honey, Sugar
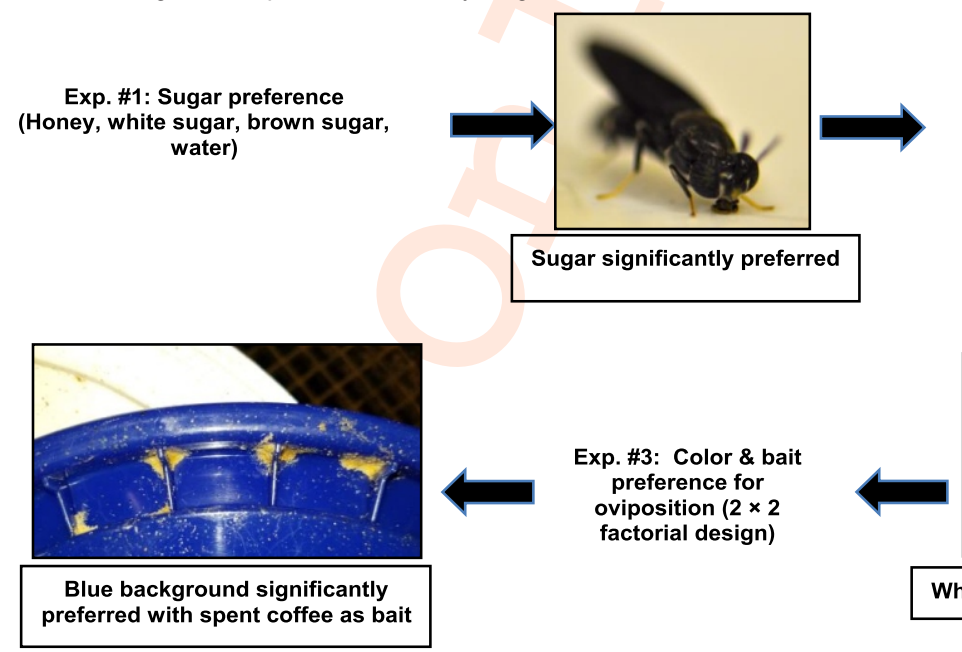

Exp. \#2: Color preference for feeding (white, brown, yellow, green, blue \& pink)

Exp. \#3: Color \& bait preference for oviposition $(2 \times 2$ factorial design)

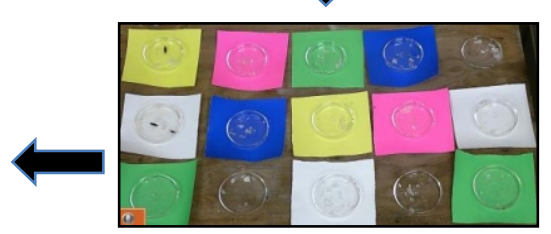

White significantly preferred and blue avoided

How to cite : Romano, N., H. Fischer and N. Egnew: Color and sugar preferences of adult black soldier fly (Hermetia illucens) (Dipetera: Stratiomyidae) for feeding and oviposition. J. Environ. Biol., 41, 1132-1137 (2020). 


\section{Introduction}

There is an increasing focus on sustainable farm practices and the black soldier fly, Hermetia illucens, has many beneficial characteristics conducive to this goal (Romano, 2018). In terms of production, $H$. illucens adults can easily reproduce under captive conditions using simple and inexpensive equipment (Tomberlin and van Huis, 2020). Moreover, their larval stages can thrive on various organic materials, either suitable or unsuitable for human consumption, such as livestock manure, organic leachates, fruits and vegetables, abattoir waste, grains and general kitchen/grocery waste (Sheppard et al., 1994; Sheppard et al., 2002; Popa and Green, 2012; Nguyen et al., 2015). The type of feed influences the nutritional content of larvae, but they are generally rich in proteins and lipids (Lalander et al., 2019). Thus, there is great potential to improve sustainable farming by transforming unwanted agricultural and food waste into a high quality feedstuff for various farmed animals (Smetana et al., 2019). In particular, the supplemental inclusion of $H$. illucens larvae to poultry diets improve chicken egg productivity and weights (Park et al., 2017) as well as broiler growth (Hwangbo et al., 2009). Moreover, there has been remarkable progress on improving the feasibility of replacing unsustainable marine-based products with $\mathrm{H}$. illucens larvae in the diets of some farmed fish species (Belghit et al., 2019, Wang et al., 2019; Fawole et al., 2020). Further, to the biodiesel industry due to high fat content (Wong et al., 2019).

The remarkable potential of using $H$. illucens larvae in different industries has yielded research into evaluating different culture systems (Sheppard et al., 2002; Nakamura et al., 2015; Ewusie et al., 2019; Hoc et al., 2019), as well as determining optimal humidity conditions, lighting, temperatures and nutrition to improve adult $H$. illucens mating and oviposition (Tomberlin and Sheppard, 2002, Zhang et al., 2010, Holmes et al., 2012). In the case of the latter, it is sometimes anecdotally reported that $H$. illucens adults cannot feed. This is despite the provision of water and/or sugar being encouraged decades ago (Furman et al. 1959), as well as their mouthparts being described (Rozkošny, 1982). Subsequently, Tomberlin et al. (2002) found that allowing $H$. illucens adults some access to water can substantially extend their lifespan. More recently, Bruno et al. (2019) provided a detailed histological and ultrastructural description of mouthparts and digestive system of $H$. illucens adults as well as demonstrated that providing a sugar solution more than doubled their lifespan. Sugar solutions were also shown to increase egg production in adult $H$. illucens females compared to water or meat broth (Lupi et al., 2019). These findings are certainly consistent with adult $H$. illucens often being observed to feed on honeydew and pollen in nature (Oldroyd, 1973, Beuk, 1990). Better results were observed with a peptone solution, over sugar or milk powder solutions, on the survival rates and egg production in adult $H$. illucens (Bertinetti et al., 2019). Whether providing water, sugar or a protein rich solution, there are clear longevity and reproductive benefits of allowing adults access to water or nutrient sources compared to nothing.

There are still some knowledge gaps regarding feeding and oviposition preferences, and research in these areas could potentially be helpful towards optimizing larval production and thus sustainable farming practices. The aim of this study was to determine the preference of adults $H$. illucens to different sugar types, followed by examining which color background encourages the feeding. The most and least preferred colors for feeding, in combination with three different bait types, were used to examine these factors on the oviposition of $H$. illucens adults.

\section{Materials and Methods}

Insect colony : Advanced $H$. illucens larvae purchased from a local supplier (Josh's Frogs, Owosso, MI) were cultured indoor on a mixture of pizza dough, fruits and spent coffee grounds on a vertically stacked compost structure. The temperature and relative humidity in the room was maintained at $31^{\circ} \mathrm{C}$ and $45 \%$, respectively, to provide favourable conditions for the larvae and adult flies (Holmes et al., 2012). Temperature was controlled using a warm air blower while humidity was increased with a humidifier as well as manually turning on a mister each day. The photoperiod was $24 \mathrm{hr}$ of light from overhead fluorescent lights at 342-586 lux. Adults hatched after four weeks of initially adding the larvae and within two days the first experiment was conducted on sugar, followed by the color preference studies. The adults were not enclosed and allowed to move freely the room. Thus, the sex ratio was not determined.

The adult $H$. illucens have tendency to get trapped in liquids and drown, particularly if the liquid is sticky. Therefore, in both the experiments, sugar was placed on one side of the petri dish and water was sprayed twice. In this way, there were dry areas for the adults to land/move safely.

Sugar preference for feeding: Water (control), white sugar, brown sugar and honey that were placed in a glass petri dish (100 $\times 15 \mathrm{~mm}$ ). For the white and brown sugar, two grams were added, while for honey, two drops were added, which was approximately one gram. After adding these sugar types, one spray from a water bottle was added to each petri dish. Each treatment was triplicated and the replicates were randomly arranged on a white poster board. Above the petri dishes was a game camera (Bushnell CORE ${ }^{\mathrm{TM}} \mathrm{HD}$ camera) that was programmed to take pictures at five min intervals over five days. The images were downloaded and the presence of flies was manually counted.

Color preference for feeding: For colour preference experiment, yellow, pink, brown, green, white and blue poster boards were cut into $11 \times 11 \mathrm{~cm}^{2}$ pieces. Each glass petri dish was placed on top of each square. Sugar with water, as described 


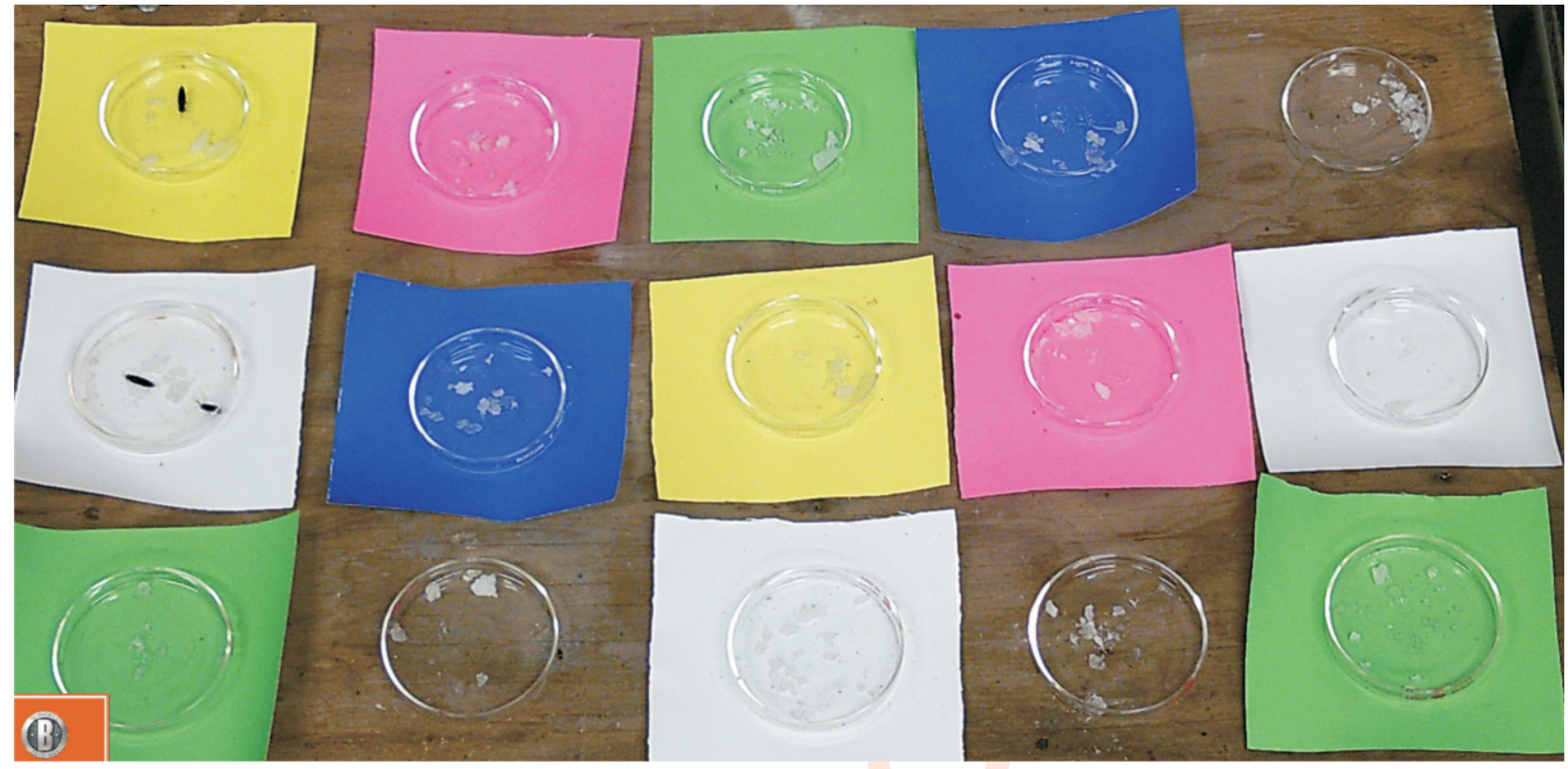

Fig. 1 : Experimental set-up showing most of the replicates in the color preference experiment.

above, was added in equal amount to each petri plate. The experiment was carried out in triplicate and arranged randomly (Fig. 1). Pictures were taken at an interval of 5 min for four days and the presence of adult flies was counted manually.

Color and bait preference for oviposition : There were two main factors that included the bait type, which included spent coffee grounds, overripe sweet potatoes and spoiled fish feeds, with the other main effect being different colors that included white or dark blue corrugated plastic lids with numerous crevices. There was a total of $2.2 \mathrm{~kg}$ of each food type, which were divided among the three replicates and covered in mosquito netting. These bait types were chosen based on local availability at no cost and the products would have been thrown away otherwise. The sweet potatoes were cut into approximately one inch cubes. The fish feeds were designed for salmonids (crude protein $55 \%$ and crude lipid $8 \%$ ) and were considered spoiled based on a slightly rancid smell and extensive fungal growth. The spent coffee was supplied from a local coffeehouse. After adding all the baits, equal amount of distill water was added to each container (height, width and diameter of 14.1,11.9 and 11.9 inches, respectively).

Each platform was placed vertically inside the container to allow adults access each side. All containers were arranged randomly and there were a total of three replicates for each treatment. At five-day interval, for up to 15 days, the number of clutches was recorded, removed using a scalpel and weighed. Due the damage sustained by the removal of eggs, hatchability was not assessed.

Statistical analyses : For Experiments 1 and 2, the total number of observations on different sugar types or colors was averaged from each replicate. The data was subjected to normality and homogeneity of variance tests. One-way ANOVA was used to determine significant differences, and difference among treatments were identified by a Tukey's post-hoc test. For Experiment 3, the total amount of clutches, and total weights and average individual clutch weights over 15 days were combined and subjected to two-way ANOVA with the main effects being color and bait type. Tukey's post-hoc test was used to determine any difference among the bait types. In order to identify differences among all the color/bait type treatments. One-way ANOVA was used followed by Tukey's post hoc test.

\section{Results and Discussion}

Sugar preference for feeding : After 5 days, 341 adults of $H$. illucens were observed on the petri dishes. Out of these, the majority were present on honey treatment, which was significantly higher $(p<0.05)$ compared to water, white sugar or brown sugar treatments (Fig. 2).

Color preference for feeding : After 4 days, 503 adults of $H$. illucens were present on the petri dishes. Only one fly was observed on blue color feeding while the highest number of flies were observed on the white color (Fig. 3), and these treatments were significantly different from all others $(p<0.05)$. The second highest number of flies were observed on the green color, which was significantly different than the yellow, brown, pink and blue treatments.

Both bait type and color significantly influenced the clutch weight and number of clutches $(p<0.05)$ (Fig. 4a,b). Dark blue 


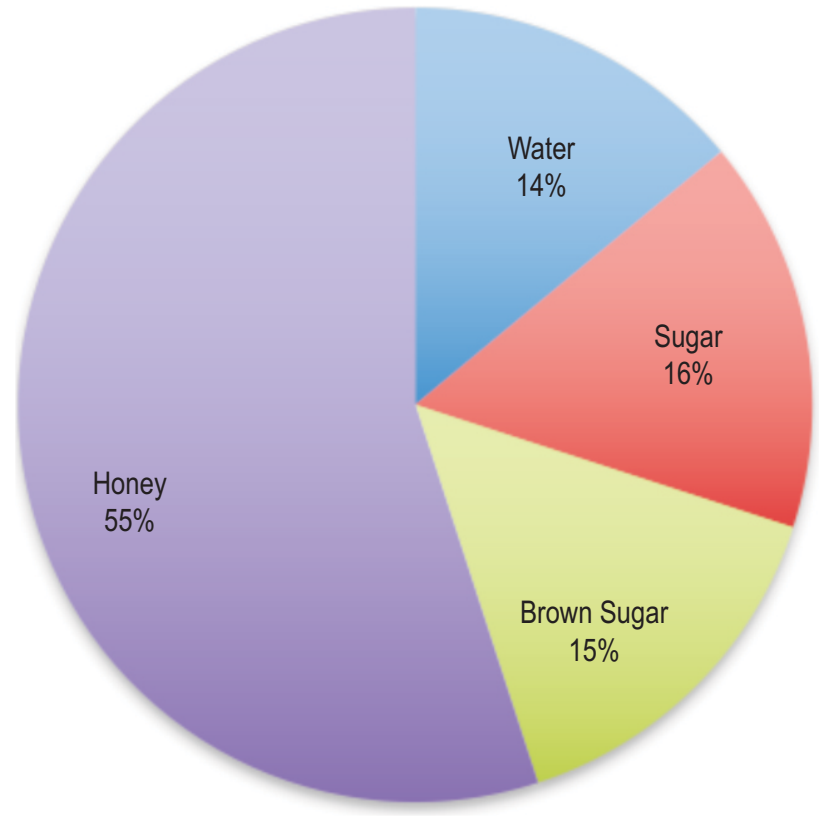

Fig. 2 : Percentage preference of adult Hermetia illucens to different sugar types and water.

color led to significantly more clutches and clutch weights compared to white color. Among the different baits, the spent coffee grounds had significantly higher amount of clutches and clutch weights. There was a significant interaction between bait type and surface color on both clutch weight and number of clutches.

The life span of adult $H$. illucens is relatively short, i.e., 415 days if they are not provided with water or nutrition (Bertinetti et al., 2019). Sugar is known to extend the life span of $H$. illucens by another week and is an effective way to improve their larval production (Lupi et al., 2019). Sugars are natural nutritional source for adult $H$. illucens, but different type of sugars have not yet been investigated in terms of preference. In the first experiment, honey was overwhelmingly preferred compared to water, white sugar or brown sugar. In fact, there was no observable preference among the water, white sugar or brown sugar treatments. However, the implications of $H$. illucens adults feeding on honey to their life span and/or oviposition needs to be further investigated, particularly considering honey contains a more diverse mixture of disaccharides and minerals as well as more calories than sugar (Ball, 2007). Based on these findings, the use of honey may be especially advantageous when trying to attract and catch wild $H$. illucens adults to potentially increase genetic diversity over time as well as starting a new colony.

In terms of feeding, there were significantly more adult $H$. illucens choosing a white coloration when sugar was provided. But perhaps more striking was that the adults appeared to

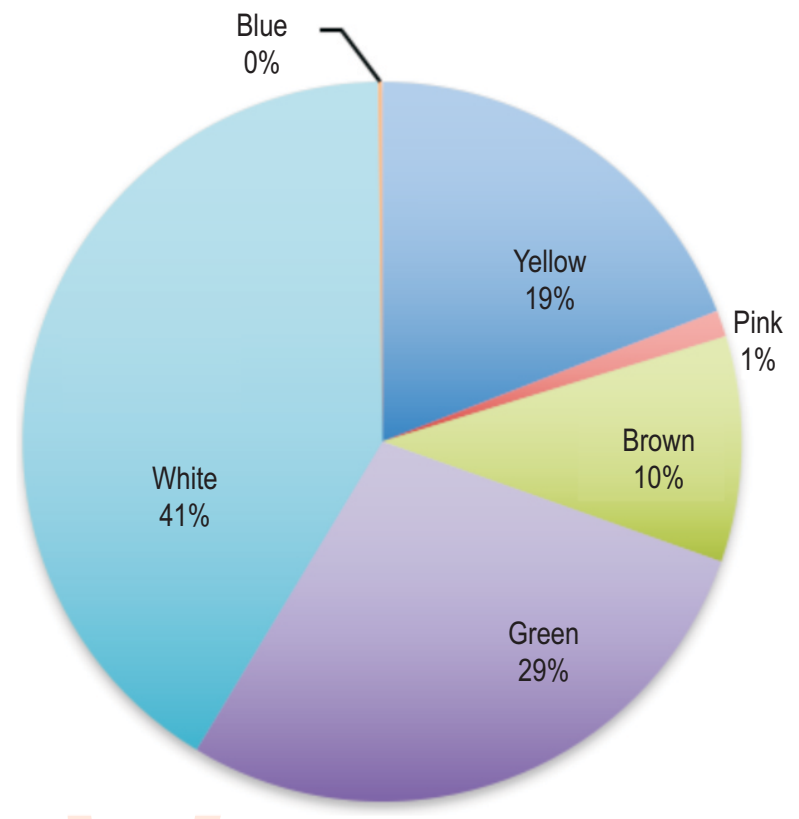

Fig. 3 : Percentage preference of adult Hermetia illucens to different colors.

completely avoid the blue color. It is important to note that the blue coloration was not dark enough to obscure the counting of the adults and potentially bias the data. There is limited information on color preference in flies. However, in a recent study, Drosophila spp. avoided blue lighting (Lazopulo et al., 2019). Undoubtablely, the mechanisms would be different than this study, which used different colored backgrounds that would unlikely influence the eye receptors of the adults as demonstrated by Lazopulo et al. (2019) when using different colored lighting.

Interestingly, when taking the best and worst treatments for feeding, of white and blue, respectively, the results to oviposition were completely the opposite as the feeding preference experiment. Unexpectedly, the blue colored platforms led to significantly more egg clutches and weights compared to the white platforms. This behavioral difference among studies may indicate that $H$. illucens adults avoid eating and laying eggs in the same area, but this should receive additional investigations.

Among the different baits, spent coffee led to significantly more egg clutches and weights compared to either the sweet potato or fish feeds. This was also unexpected considering $H$. illucens larvae have lower growth on spent coffee compared to other organic substrates (Lardé, 1990; Permana et al., 2018). Research should investigate the potential of fungal toxins (e.g. deoxynivalenol, zearalenone, and aflatoxins) and oxidized lipids to deter oviposition in $\mathrm{H}$. illucens. However, it is important to note that the fish feeds along with sweet potato attracted other fly species, including Drosophila spp., and Musca domestica that 

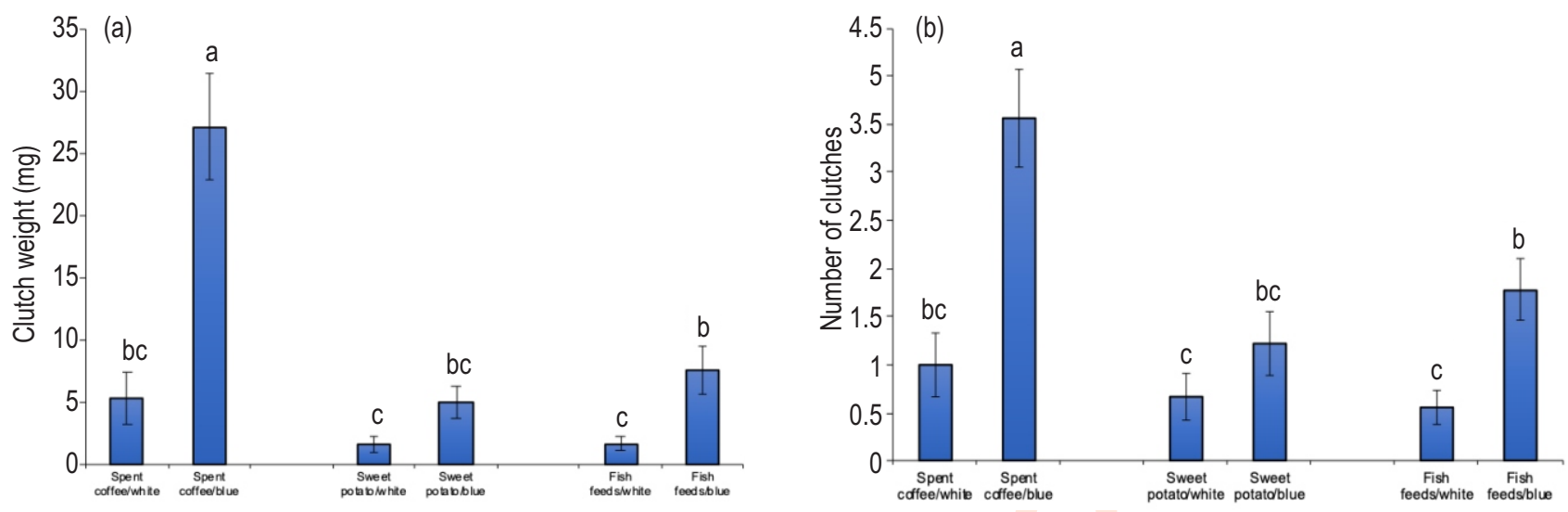

Fig. $4(\mathrm{a}, \mathrm{b})$ : The mean $( \pm S E)$ clutch weights $(\mathrm{mg})(\mathrm{a})$ and number of clutches $(\mathrm{b})$ on dark blue versus white platforms with different bait types.

were present in the room and laid eggs. The presence of adult and eggs of other fly species may have deterred the adult $H$. illucens from the extra competition. For example, Miranda et al. (2019) showed that when $M$. domestica already inhabited chicken manure, this deterred the oviposition of $H$. illucens adults. Subsequent to this study, it became standard practice in our lab to layer spent coffee grounds on top of different food types along with adding some $\mathrm{H}$. illucens larvae to potentially deter other fly species, with the latter consistent with the findings of Furman et al. (1959).

The obtained results can provide some cost-effective and practical ways to improve $H$. illucens larval production. Taken together, it is suggested that providing honey on a white background would encourage the feeding of $H$. illucens adults while providing a dark blue substrate excluded feeding but encouraged oviposition compared to a white background. This type of compartmentalization could better facilitate the feeding and breeding/egg collections of $H$. illucens adults. The implications of these practices on the life span and fecundity of $H$. illucens adults should be further investigated as well as other ways to enhance feeding/oviposition, such as different olfactory stimulants. Such studies should help optimize their production and, in turn, sustainable farming practices.

\section{Acknowledgments}

This study was funded from a National Institute of Food and Agriculture (NIFA) Capacity Building Grant (award \#: 201938821-29047).

\section{References}

Ball, D.: The chemical composition of honey. J. Chem. Educ., 84, 10 (2007).

Belghit, I, N.S. Liland, P. Gjesdal, I. Biancarosa, E. Menchetti, Y. Li, R. Waagb $\varnothing$, A. Krogdahl and E.J. Lock: Black soldier fly larvae meal can replace fish meal in diets of sea-water phase Atlantic salmon
(Salmo salar). Aquaculture, 503, 609-619 (2019).

Bertinetti, C., A.C. Samayoa and S.Y. Hwang: Effects of feeding adults of Hermetia illucens (Diptera: Stratiomyidae) on longevity, oviposition, and egg hatchability: Insights into optimizing egg production. J. Insect. Sci., 19, 1-7 (2019).

Beuk, P.L.T.: Honeydew as a food source for insects and in particular for soldier flies (Diptera: Stratiomyidae). Phegea, 18, 137-140 (1990).

Bruno, D., M. Bonelli, A.G. Cadamuro, M. Reguzzoni, A. Grimalidi, M. Casaterlli and G. Tettamanti: The digestive system of the adult Hermetia illucens (Diptera: Stratiomyidae): Morphological features and functional properties. Cell Tiss. Res., 378, 221-238 (2019).

Ewuise, E.A., P.K. Kwapong, G. Ofosu-Budu, C. Sandrock, A.M. Akumah, E.K. Nartey, C. Tetegaga and S.K. Agyakwah: The black soldier fly, Hermetia illucens (Diptera: Stratiomyidae): Trapping and culturing of wild colonies in Ghana. Scien. Afr., 5, e00134 (2019).

Fawole, F.J., AA. Adeoye, L.O., Tiamiyu, K.I. Ajala, S.O. Obadara and I.O. Ganiyu: Substituting fishmeal with Hermetia illucens in the diets of African catfish (Clarias gariepinus): Effects on growth, nutrient utilization, haemato-physiological response, and oxidative stress biomarker. Aquaculture, 518, 734849 (2020).

Fischer, $\mathrm{H}$. and N. Romano: Black soldier fly larval production: Development, testing of all-in-one, stacked production system. Glob. Aquacult. Advo. March (2020).

Furman, D., R. Young and P. Catts: Hermetia illucens (Linnaeus) as a factor in the natural control of Musca domestica Linnaeus. J. Econo. Entomol., 52, 917e921 (1959).

Hoc, B., G. Noël, J. Carpentier, F. Francis and R.C. Megido: Optimization of black soldier fly (Hermetia illucens) artificial reproduction. PLOS ONE, 14, e0216160 (2019).

Holmes, L.A., S.L. Vanlaerhoven and J.K. Tomberlin: Relative humidity effects on the life history of Hermetia illucens (Diptera: Stratiomyidae). Environ. Entomol., 41, 971-978 (2012).

Hwangbo, J., E.C. Hong, A. Jang, H.K. Kang, J.S. Oh, B.W. Kim and B.S. Park: Utilization of house fly-maggots, a feed supplement in the production of broiler chickens. J. Environ. Biol., 30, 609-614 (2009).

Lardé, G.: Recycling of coffee pulp by Hermetia illucens (Diptera: 
Stratiomyidae) larvae. Biolog. Wast., 33, 307-310 (1990).

Laxopulo, S., A. Lazopulo, J.D. Baker and S. Syed: Daytime colour preference in Drosophila depends on the circadian clock and TRP channels. Nature, 574, 108-111 (2019).

Lupi, D., S. Savoldelli, M.G. Leonardi and C. Jucker: Feeding in the adult of Hermetia illucens (Diptera: Stratiomyidae) reality or fiction? J. Entomolog. Acraolog. Res., 51, 8046 (2019).

Miranda, C.D., J.A. Cammack and J.K. Tomberlin: Interspecific competition between the house fly, Musca domestica L. (Diptera: Muscidae) and black soldier fly, Hermetia illucens (L.) (Diptera: Stratiomyidae) when reared on poultry manure. Insects, 10, 440 (2019).

Nakamura, S., R.T. Ichiki, M. Shimoda and S. Morioka: Small-scale rearing of the black soldier fly, Hermetia illucens (Diptera: Stratiomyidae), in the laboratory: low-cost and year-round rearing. Appl. Entomol. Zool., 51, 161-166 (2016).

Nguyen, T.T.X., J.K. Tomberlin and S. Vanlaerhoven: Ability of black soldier fly (Diptera: Stratiomyidae) larvae to recycle food waste. Environ. Entomol., 44, 406-410 (2015).

Oldroyd, H.: Diptera brachycera. section (a) tabanoidea and asiloidea. Handbk. Ident. Br. Insects IX, 4, 10-14 (1973).

Park, B.S., K.H. Um, W.K. Choi and S.O. Park: Effect of feeding black soldier fly pupa meal in the diet on egg production, egg quality, blood lipid profiles and faecal bacteria in laying hens. Europ. Poult. Sci., 81 (2017).

Permana, A.D. and J.E.N.R.E. Putra: Growth of black soldier fly (Hermetia illucens) larvae fed on spent coffee ground. IOP Conf. Series: Earth Environ. Sci., 187, 012070 (2018).

Popa, R. and T.R. Green: Using black soldier fly larvae for processing organic leachates. J. Econo. Entomol., 105, 374-378 (2012).

Romano, N.: Alternative and new sources of feedstuffs. In: Enzymes in Human and Animal Nutrition: Principles and Perspectives (Eds.:
C.S. Nunes and V. Kumar). Academic Press, Elsevier. pp. 381-396 (2018).

Smetana, S., E. Schmitt and A. Mathys: Sustainable use of Hermetia illucens insect biomass for feed and food: Attributional and consequential life cycle assessment. Res. Conserv. Recy., 144, 285-296 (2019).

Sheppard, D.C., G.L. Newton, S.A. Thompson and S. Savage: A value added manure management system using the black soldier fly. Biores. Technol., 50, 275-279 (1994).

Sheppard, D.C., J.K. Tomberlin, J.A. Joyce, B.C. Kiser and S.M. Sumner: Rearing methods for the black soldier fly (Diptera: Stratiomyidae). J. Med. Entomol., 39, 695-698 (2002).

Tomberlin, J.K., D.C. Sheppard and J.A. Joyce: Selected life history traits of the black soldier fly. Ann. Entomolog. Soc. Amer., 95, 379-386 (2002).

Tomberlin, J.K. and D.C. Sheppard: Factors influencing mating and oviposition of black solider flies (Diptera: Stratiomyidae) in a colony. J. Entomol. Sci., 37, 345-352 (2002).

Tomberlin, J.K. and A. van Huis: Black solider fly from pest to 'crown jewel' of the insects as feed industry: An historical perspective. J. Insect. Food Feed, 6, 1-4 (2020).

Wang, G., K. Peng, J. Hu, C. Yi, X. Chen, H. Wu and Y. Huang: Evaluation of defatted black soldier fly (Hermetia illucens L.) larvae meal asan alternative protein ingredient for juvenile Japanese seabass (Lateolabrax japonicus) diets. Aquaculture, 507, 144-154 (2019).

Wong, C.Y., S.S. Rosli, Y. Uemura, Y.C. Ho, A. Leejeerajumnean, W., Kiatkittipong, C.K. Cheng, M.K., Lam and J.W. Lim: Potential protein and biodiesel sources from black soldier fly larvae: Insights of larval harvesting instar and fermented feeding medium. Energies, 12, 1570 (2019).

Zhang, J., L. Huang, J. He, J.K. Tomberlin, J. Li, C. Lei, M. Sun, Z. Liu and Z. Yu: An artificial light source influences mating and oviposition of black soldier flies, Hermetia illucens. J. Insect Sci., 10, 202 (2010). 